\title{
Análisis cuantitativo de sistemas de fracturas y sus implicaciones hidrogeológicas en la zona sur de la Mesa de Los Santos, Santander - Colombia
}

\author{
Yessenia Tarazona-Lizcano $^{1^{*}}$ (D); María Camila Vargas-López ${ }^{1}$ (D); Francisco Velandia ${ }^{1}$
}

Forma de citar: Tarazona-Lizcano, Y.; Vargas-López, M.C.; Velandia, F. (2021). Análisis cuantitativo de sistemas de fracturas y sus implicaciones hidrogeológicas en la zona sur de la Mesa de Los Santos, Santander Colombia. Boletín de Geología, 43(3), 107-123. https://doi.org/10.18273/revbol.v43n3-2021005

\begin{abstract}
Resumen
El análisis cuantitativo del sistema de fracturas emplea métodos y procesamientos estadísticos para determinar la geometría de las estructuras y parámetros como intensidad, densidad y longitud de fractura con el fin de identificar las zonas más favorables para la infiltración y almacenamiento de fluidos en un área específica. Para llevar a cabo este análisis en la zona sur de la Mesa de Los Santos se empleó el software FracPaQ, el cual mide y cuantifica patrones de fractura en dos dimensiones a partir de datos digitales obtenidos de fotografías de afloramientos; estos datos son mostrados en mapas de rangos y de dispersión para correlacionarlos con atributos geológicos registrados en cada estación. Se presenta también un análisis preliminar del flujo de fluidos en gráficos de conectividad y permeabilidad. Esta cuantificación permitió definir que las fallas NW-SE destacan en el arreglo espacial y por la apertura de las fracturas son potenciales corredores para la circulación de fluidos al sureste de la Mesa de Los Santos. Se propone la zona influenciada por la Falla La Mojarra como un sector de alta porosidad secundaria favorable para la infiltración y flujo subsuperficial asociado al miembro superior de la Formación Los Santos.
\end{abstract}

Palabras clave: Análisis estadístico; Patrones de fractura; FracPaQ; Ventanas circulares; Flujo de fluidos.

\section{Quantitative analysis of the fracture systems and groundwater implications in the south zone of the Mesa de Los Santos, Santander - Colombia}

\begin{abstract}
The quantitative analysis of the fracture system is the use of statistical methods and procedures to determine the structures geometry and the parameters that describe the fracturing such as intensity, density, and fracture length with the purpose of identifying the most favorable areas for infiltration and fluid storage in a specific area. To implement this analysis in the south of the Mesa de Los Santos, the FracPaQ software was used, which measures and quantifies fracture patterns in two dimensions based on digital data obtain from outcrops photograph. This data is shown in range and dispersion maps to correlate with geologic attributes taken from each station. As additional information, a preliminary analysis of the flow of fluids is presented in graphs of connectivity and permeability in the direction of the flow. This quantification allowed establishing that the NW-SE faults stand out in the spatial arrangement and opening of the fractures, acting as the main corridors for the circulation of fluids in the southeast of the Mesa de Los Santos. We propose the zone influenced by the La Mojarra Fault as a sector of high secondary porosity favorable for infiltration and subsurface flow associated with the upper member of the Los Santos Formation.
\end{abstract}

Keywords: Statistical analysis; Fracture patterns; FracPaQ; Circular windows; Fluid flow.

${ }^{1}$ Grupo de Investigación en Geología Básica y Aplicada, Escuela de Geología, Universidad Industrial de Santander, Bucaramanga, Colombia. (*) yesenara95@gmail.com; mariac_0603@hotmail.com; favelanp@uis.edu.co 


\section{Introducción}

La geología estructural es una de las disciplinas fundamentales en los estudios hidrogeológicos ya que permite la obtención de información y análisis de patrones de fractura en los sistemas acuíferos. Con criterios aplicados es posible reconocer y proponer potenciales canales de circulación y almacenamiento del recurso hídrico asociados a la porosidad secundaria en las unidades geológicas de una zona.

La Mesa de Los Santos o de Xerira (palabra Guane) es un área de aproximadamente $430 \mathrm{~km}^{2}$ que comprende los municipios de Los Santos, Girón y Piedecuesta (Santander). Fisiográficamente se localiza en el costado oeste de la Cordillera Oriental. Para el propósito de esta investigación se delimitó un área de estudio al sur de la mesa caracterizada geomorfológicamente por presentar planicies, colinas denudadas, laderas de contrapendiente de cuesta y estructurales de cuesta, onduladas, erosivas y zonas de pendientes muy abruptas hacia los bordes de la Mesa (Moreno y Silva, 2021). El área abarca el casco urbano de Los Santos y las veredas aledañas cuya calidad de vida de los habitantes ha sido más afectada por la problemática de escasez de agua.

La Mesa de Los Santos ha sido objeto de diversos estudios hidrogeológicos donde la escasez de agua obliga a buscar alternativas para el abastecimiento como el uso de aguas subterráneas. INGEOMINAS (actual Servicio Geológico Colombiano) lideró la exploración hidrogeológica en convenios con la Universidad Industrial de Santander (UIS) (Pinto et al., 2007), incluyendo el apoyo para trabajos de grado de las escuelas de Geología e Ingeniería Civil (Vargas, 2008; Contreras, 2008; Arguello et al., 2018). De la zona también se conocen informes de consultoría para la explotación del recurso hídrico. Estas investigaciones han dado a conocer la problemática de la mesa, donde aún no se cuenta con una solución para la escasez y suministro, especialmente en la zona sur. El presente trabajo aporta y actualiza información de la cantidad de fracturas de la zona sur de la Mesa de Los Santos con el fin de definir posibles sectores favorables para la infiltración y almacenamiento. Así mismo, se establecen las orientaciones de las familias de diaclasas principales y se realiza un análisis preliminar de flujo de fluidos al sistema acuífero. Estos resultados tienen implicaciones hidrogeológicas importantes para la construcción de un modelo conceptual y el estudio de aguas subterráneas en la Mesa de Los Santos al caracterizar los potenciales corredores de flujo.

Esta investigación siguió una metodología sencilla donde se usaron fotografías de campo digitalizadas en un programa de procesamiento estadístico llamado FracPaQ (Healy y Rizzo, 2019) para conocer los atributos de los sistemas de fractura (orientaciones preferenciales, apertura, intensidad, densidad, longitud promedio, conectividad y permeabilidad), cuyo análisis aporta a la caracterización de potenciales corredores de flujo. En este sentido, el trabajo muestra algunos de los resultados de la investigación de Tarazona y Vargas (2020), la cual hace parte del proyecto "Estudio integral del agua en la Mesa de Los Santos", financiado por la UIS.

\section{Marco geológico}

La Mesa de Los Santos se encuentra ubicada dentro de la zona tectonoestratigráfica central del departamento de Santander y pertenece al área de bloques hundidos conformados por rocas de edades del Mesozoico y Cenozoico (Clavijo y Royero, 2001). El basamento se compone de rocas metamórficas de bajo a medio grado del Paleozoico pertenecientes a la Formación Silgará y rocas ígneas intrusivas del Jurásico pertenecientes al Granito de Pescadero. La secuencia sedimentaria inicia con rocas continentales del Jurásico de la Formación Jordán, seguidas de rocas del Cretácico de las formaciones Los Santos, Rosablanca, La Paja y Tablazo. También se encuentran localmente depósitos no consolidados del Cenozoico (Figura 1).

La Formación Los Santos conformada por tres niveles, presenta un espesor total de más de 200 $\mathrm{m}$ en el borde oeste de la Mesa de Los Santos y de $120 \mathrm{~m}$ en el borde este, es decir que se adelgaza hacia el Macizo de Santander (Pinto et al., 2007); El nivel superior es de principal interés hidrogeológico $\mathrm{y}$ se encuentra constituido por areniscas cuarzosas con variaciones granulométricas de grano medio a muy fino y diferentes grados de compactación. La Formación Rosablanca cuyo espesor varía entre 150 y 425 m (Pinto et al., 2007) está constituida por litologías calcáreas y evaporíticas en capas de espesor variable, principalmente secuencias de capas de caliza intercaladas con yeso, margas y en ocasiones lodolitas calcáreas (Figura 1). 


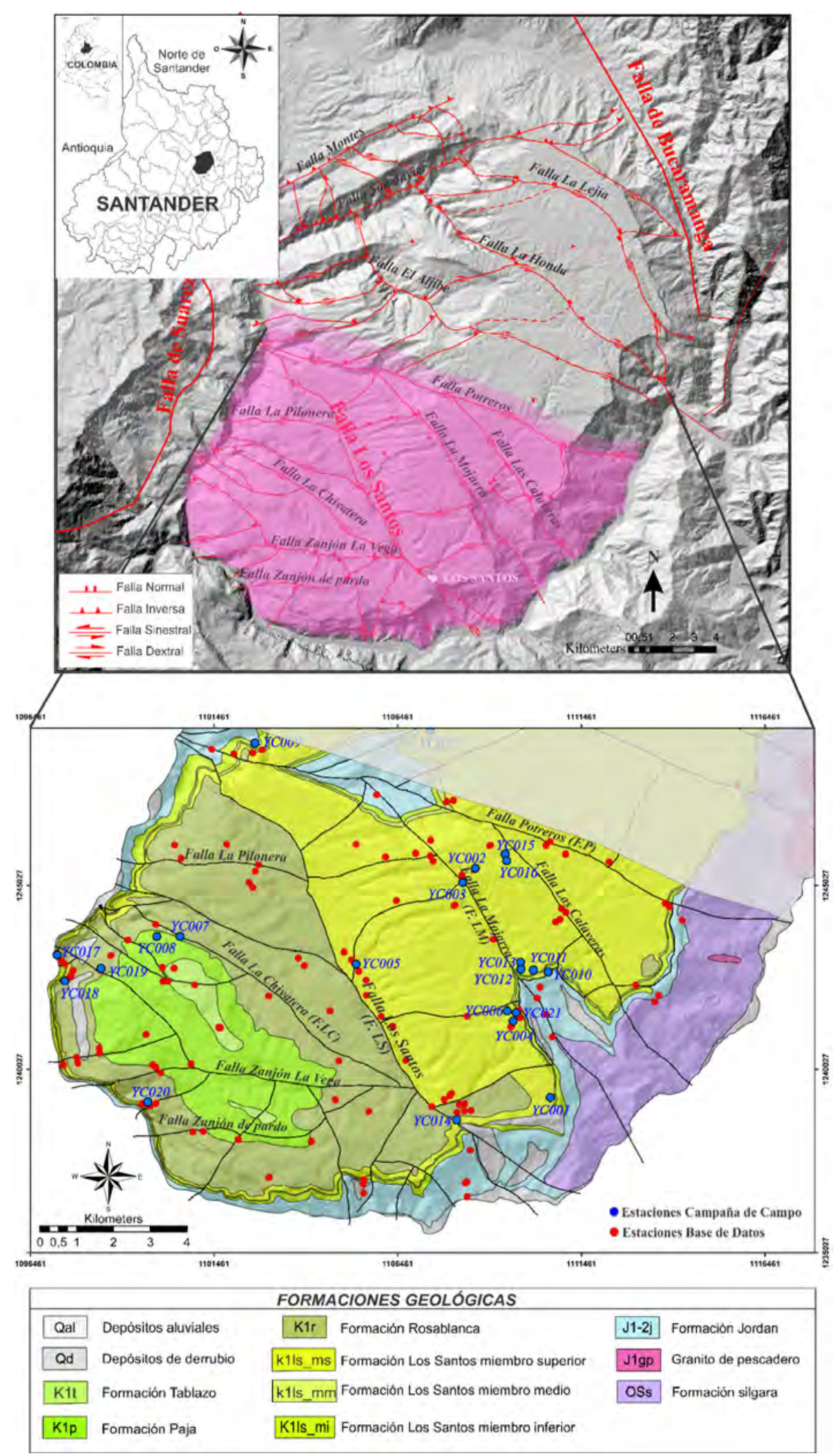

Figura 1. Localización del área de estudio (sombra rosada). El mapa superior muestra el marco estructural regional alrededor de la zona de estudio. El mapa inferior muestra la geología, estaciones y fallas de la zona sur de la Mesa de Los Santos. Modificado de Pinto et al. (2007). 
Estructuralmente la Mesa de Los Santos se encuentra localizada entre las fallas de Suárez (al oeste) y Bucaramanga (al este), de las cuales se reporta actividad reciente $<1,6$ Ma (Cuaternario), con movimiento inversos y sinestrales (París et al., 2000). Julivert (1958) reporta evidencias en la reorganización de la red de drenaje, especialmente por eventos de captura por corrientes piratas que dieron lugar a los actuales cauces de los ríos Chicamocha y Sogamoso que a su vez delimitaron el actual paisaje de mesas y cuestas. La Falla Suarez corresponde a una falla inversa de alto ángulo que sigue el curso de los ríos Suarez y Sogamoso (Pinto et al., 2007) y la Falla de Bucaramanga es una estructura de tipo regional con componente combinado en el rumbo (sinestral) y en el buzamiento (inverso) (Taboada et al., 2000; Velandia y Bermúdez, 2018; Siravo et al., 2020) (Figura 1). La Falla de Los Santos es la estructura que afecta principalmente la zona de estudio, y es mostrada por Velandia et al. (2020) en forma regional al conectar y extender el nombre como Falla de Lebrija, un trazo de cinemática inversa y sinestral, subparalelo a la Falla de Bucaramanga. En la Mesa de Los Santos se distinguen fallas locales en distintas direcciones, las cuales son denominadas, por Morales et al. (2010) como sistemas longitudinales y transversales según se orienten NW-SE o NE-SW, respectivamente; además de otra tendencia con menor número de fallas de dirección W-E (Figura 1). En este marco estructural, la mayoría de fallas tienen un componente de rumbo por estar orientadas en forma oblicua a los tensores de esfuerzos en la zona (Velandia et al., 2020) y especialmente las fallas longitudinales NW han sido definidas como transtensivas y favorables para el tránsito de fluidos (Velandia et al., 2007; Vargas, 2008; Velandia, 2010).
A partir de pozos, cartografía geológica, estratigrafía, geología estructural y geología de arcillas de la zona, Pinto et al. (2007) identifican que los miembros superior e inferior de la Formación Los Santos se comportan como la principal fuente de agua subterránea en la región, mientras que el miembro intermedio actúa como sello debido a que es principalmente arcilloso. El miembro superior se recarga por alta pluviosidad, principalmente en la zona norte de la Mesa y el miembro inferior se recarga por percolación a través de fracturas y diaclasas abiertas debido al alto grado de fracturación y su alta inclinación (Pinto et al., 2007).

Las fracturas de la zona fueron estudiadas con enfoque hidrogeológico por Contreras (2008) y Díaz et al. (2009) a partir del análisis de la distribución de diaclasas abiertas y cerradas, su densidad y rangos de buzamiento, y complementan con la información de algunos de los pozos inventariados por Pinto et al. (2007), con profundidades entre $27 \mathrm{~m}$ y $150 \mathrm{~m}$. Esto les permite proponer dos sectores de interés (Figura 2): el noreste de la Mesa de Los Santos, en la vereda Holanda donde se encuentra señal de flujo a los $8,5 \mathrm{~m}$ y luego roca saturada desde los $65 \mathrm{~m}$ hasta los $85 \mathrm{~m}$; y hacia el centro de la Mesa, en las veredas El Verde, La Fuente y Tabacal, donde se identifica señales de flujo desde los $25 \mathrm{~m}$ hasta los $88 \mathrm{~m}$. Así confirman que la Formación Los Santos actúa como acuífero en el cual las fracturas permiten la recarga y se encuentran interconectadas facilitando el flujo de agua en dirección del buzamiento. La poca profundidad de algunos pozos (entre $25 \mathrm{~m}$ y $35 \mathrm{~m}$ ) muestra nuevamente que el miembro superior de la Formación los Santos es el acuífero principal y el más explotado en el área (Contreras, 2008).
A.

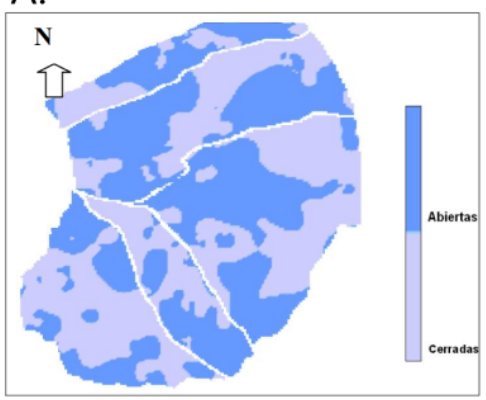

B.

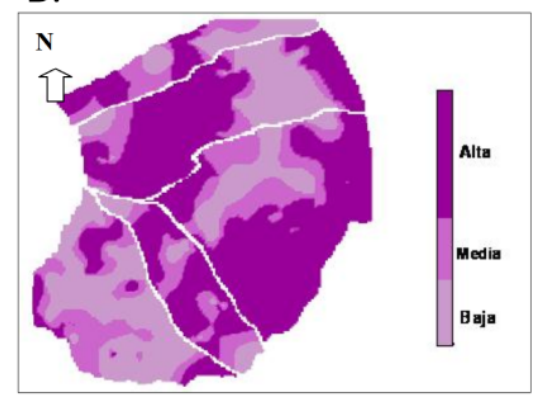

C.

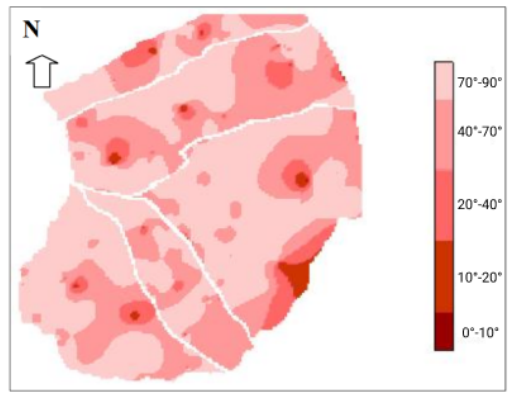

Figura 2. Mapas de distribución de la Mesa de Los Santos. A. Mapa de distribución de diaclasas abiertas y cerradas. B. Mapa de distribución espacial de la densidad de fracturamiento (cualitativo). C. Mapa de distribución espacial de los rangos de buzamiento de las diaclasas. Modificado de Contreras (2008). 


\section{Metodología}

\section{Control cartografía geológica y datos estructurales}

Como un primer acercamiento se determinó el estado actual del conocimiento en base a los trabajos de Pinto et al. (2007), Vargas (2008), Contreras (2008) y Díaz et al. (2009), lo cual permitió proponer un diagnóstico geológico e hidrogeológico de la zona. Se compiló la información de diaclasas medidas en campo de Pinto et al. (2007), se registró en una base de datos, se proyectaron en un mapa y de acuerdo a la distribución espacial mostrada se plantearon puntos estratégicos a evaluar ubicados en la zona de estudio (al sur de la Mesa de Los Santos) de tal forma que se pudiera corroborar la información de estaciones previas y visitar lugares con baja densidad de datos. La campaña de campo consistió en la medición de diaclasas en diferentes afloramientos a lo largo de la zona de estudio; para las familias de diaclasas se midió rumbo, inclinación, apertura (correspondiente a la separación de las paredes de la roca en $\mathrm{mm}$ ) y se tomaron múltiples fotografías con orientación del afloramiento y escala con el bastón de Jacob para su posterior análisis por medio del método de la ventana de muestra en el software especializado. La cámara utilizada tiene una resolución de 12MP con apertura de f/1.8mm capturadas con un iPhone XS Max.

\section{Procesamiento y análisis}

Una vez recopilada, organizada y digitalizada la información obtenida se inició su procesamiento y tratamiento estadístico utilizando los distintos programas. Mediante Georose 0.5.1 (Yong Technology Inc., 2015) se generaron diagramas de rosa para identificar la orientación preferencial del sistema de fracturas y para analizar su posible relación con las fallas principales.

Para el análisis cuantitativo de patrones de fracturas en dos dimensiones (2D) se usó FracPaQ, un software de código abierto escrito en MATLAB ${ }^{\mathrm{TM}}$ (Healy et al., 2017). Previamente y para generar el archivo de entrada se utilizó el software de diseño gráfico vectorial CorelDRAW ${ }^{\circledR}$, donde se realizó el preprocesamiento de las fotografías escogiendo la imagen que mejor representara el afloramiento, evitando la vegetación y meteorización intensa. Una vez seleccionada la fotografía de cada estación, se ingresó a Corel donde se escaló con las medidas del bastón de Jacob y sobre un rectángulo delimitador del afloramiento se dibujaron las diaclasas con la herramienta Polilínea siguiendo el camino de las fracturas visibles (Figura 3). A partir de esta entrada, FracPaQ cuantifica las longitudes y orientaciones de las fracturas constituyentes, así como la intensidad, densidad, conectividad y permeabilidad del patrón de fractura, permitiendo con estos dos últimos un análisis preliminar del flujo de fluidos (Healy et al., 2017).

Longitudes de fractura y orientaciones: las longitudes de fractura se cuantifican desde los afloramientos fotografiados con la medida a escala de la línea con la que fue trazada la fractura en CorelDRAW. Los ángulos de segmento se calculan con respecto al eje $\mathrm{Y}$, tomando como positivo el movimiento en sentido horario. En caso de que el eje Y no esté alineado hacia el norte, se permite ingresar la corrección con la respectiva rotación desde el norte. Este número se resta a cada uno de los ángulos del segmento para producir una gráfica "corregida" presentada en un diagrama de rosa.

Intensidad y densidad: la intensidad se considera como el espaciamiento promedio entre fracturas (paralelas y no paralelas) y la densidad como el número de fracturas por unidad de área. Para realizar estimaciones de estos parámetros FracPaQ emplea el método de líneas de muestreo circular y ventanas circulares de Mauldon et al. (2001) con cada uno de los rectángulos delimitadores de las imágenes digitalizadas. Esto con el propósito principal de lograr una caracterización sistemática y eficiente de los sistemas de fractura a través de relaciones matemáticas reduciendo significativamente los sesgos por orientación, censuramiento y longitud. Las bases del método de muestreo circular también se explican en Moreno-Sánchez y García-Cabrejo (2006). En FracPaQ, este análisis es llevado a cabo sobre un patrón de cuadrícula regular en el archivo de entrada utilizando el número de círculos de escaneo especificados en el cuadro de texto del programa "Number of scan circles" definiendo así el radio de la circunferencia. El valor predeterminado que aparece es 12 (Healy y Rizzo, 2019), lo que genera una cuadricula de 12x12=144 círculos de escaneo cumpliendo con la recomendación de Mauldon et al. (2001) de realizar más de 100 ventanas de muestra con medición de al menos 112 fracturas (entre otras recomendaciones). $\mathrm{Al}$ realizar varios intentos con diferentes valores el número que mejor mostraba los resultados (vistos en los mapas de calor de la Figura 4) fue este, por lo cual se estableció para nuestra investigación. El código dibuja estos círculos de exploración (Figura 4) en las direcciones $\mathrm{X}$ y Y, y cuenta las intersecciones de 
segmentos de fractura con el perímetro del círculo ( $n$ ) $\mathrm{y}$ el número de segmentos que terminan dentro del círculo $(m)$. Estas medidas se utilizan para estimar la intensidad (unidades de $\mathrm{m}^{-1}$ ) y la densidad (unidades de $\mathrm{m}^{-2}$ ) de las ecuaciones en Mauldon et al. (2001).

$$
I=\frac{n}{4 r} \quad \rho=\frac{\mathrm{m}}{2 \pi \mathrm{r} 2}
$$

$\mathrm{r}=$ radio de la circunferencia usada

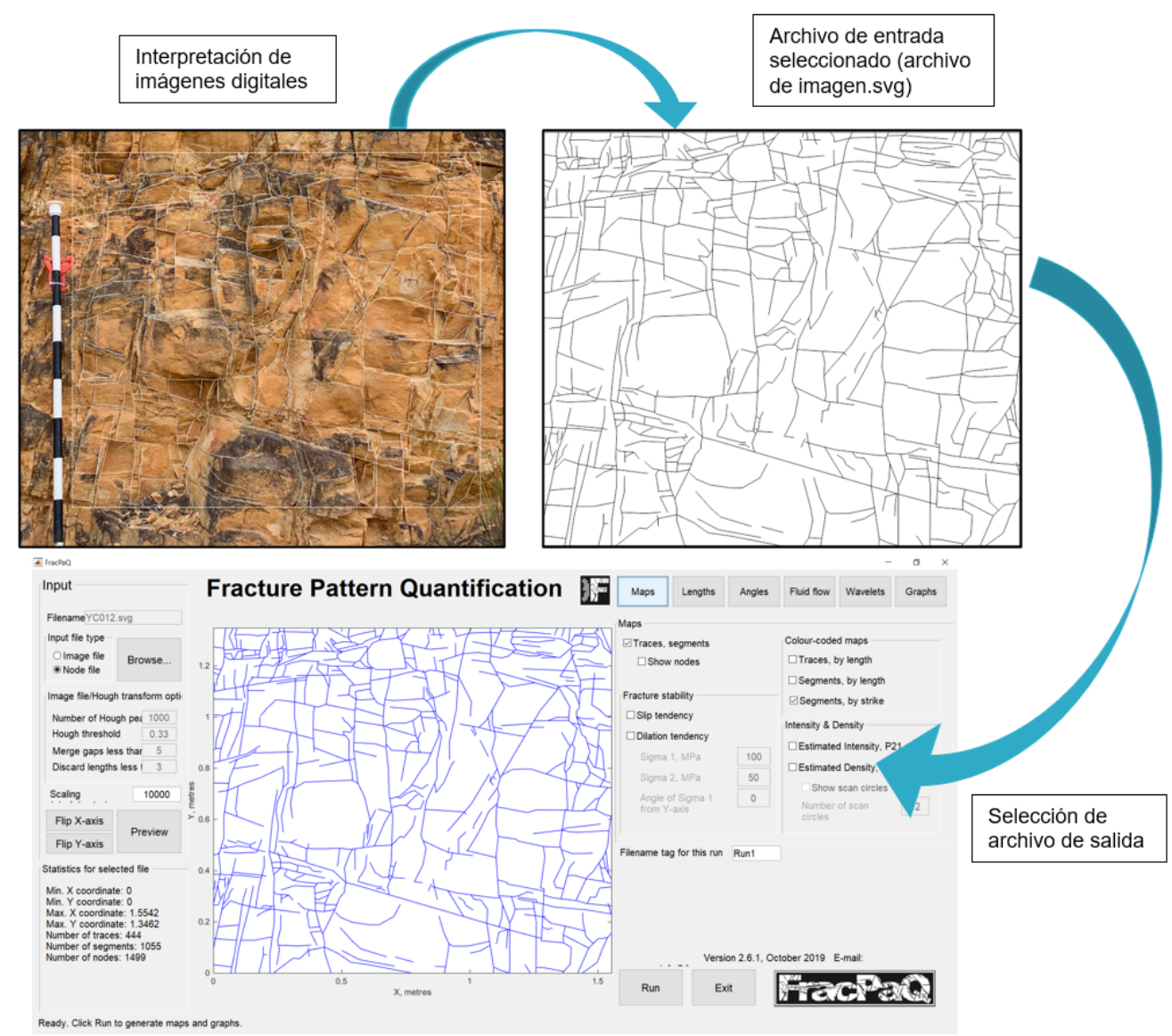

Figura 3. Metodología para llevar a cabo el análisis en el software FracPaQ, desde la fotografía de campo hasta la digitalización de trazos y cuantificación de los patrones de fractura.

A.

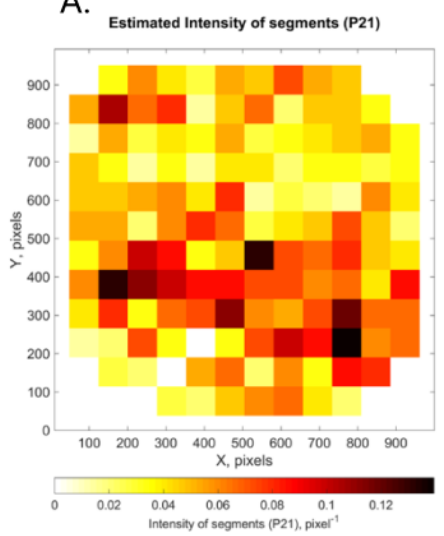

B.

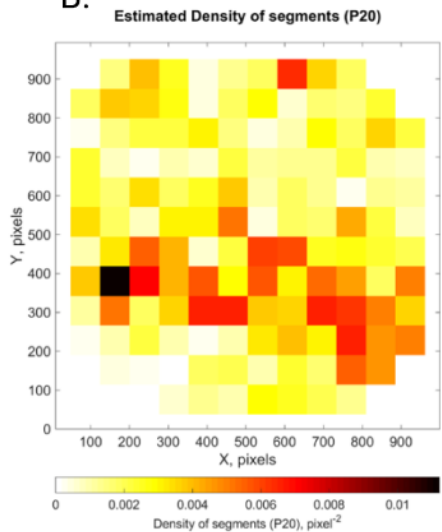

C.

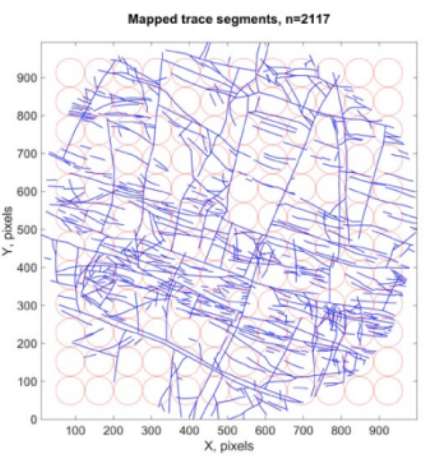

Figura 4. A. Mapa de intensidad estimada (P21, m-1). B. Mapa de densidad estimada (P20, m-2). C. Posiciones y tamaños de los círculos de exploración utilizados en las estimaciones. Tomado de Healy y Rizzo (2019). 
Conectividad y permeabilidad: la conectividad es la capacidad del sistema de fracturas para interconectarse. Este parámetro se evalúa a través de la topología, la cual describe la relación entre fracturas individuales en un sistema de fractura usando el grafico ternario de conectividad propuesto por Manzocchi (2002). El grafico cuenta con tres vértices que denota los nodos I (para extremos aislados de trazas), Y (para puntos de ramificación, aplastamientos o estribos) y X (para intersecciones transversales). De esta manera, las redes más conectadas se trazarán hacia la línea Y-X, en la parte inferior de este diagrama, mientras que las redes menos conectadas se trazarán hacia el vértice I.

Para estimar la permeabilidad de un sistema de fracturas en dos dimensiones, conocida como la capacidad que tiene un sistema de permitirle a un fluido fluir, FracPaQ utiliza la medida de la orientación preferencial de fracturas de las líneas trazadas y genera dos gráficos de anisotropía de permeabilidad, uno en dirección del flujo y otro en la dirección del gradiente de presión del fluido (Healy y Rizzo, 2019).

Los datos numéricos obtenidos a partir de FracPaQ se presentan clasificados en rangos sobre el mapa de la zona, estación por estación y de acuerdo con el parámetro estudiado. Estos valores se revisan y se discuten, dando interpretaciones al respecto según los objetivos propuestos.

\section{Resultados}

\section{Revisión de antecedentes}

El diagnóstico inicial de la situación del fracturamiento al sur de la Mesa de Los Santos se llevó a cabo con las investigaciones de Pinto et al. (2007), Contreras (2008), Vargas (2008), Díaz et al. (2009), Velandia et al. (2007), Velandia (2010) y Morales et al. (2010); las cuales fueron base para establecer una dinámica estructural de fracturamiento. Con los trabajos de Pinto et al. (2007) y Vargas (2008) se tuvo acceso a la información recolectada en campo (planos de fractura medidos de afloramientos), en la cual se encontraron algunas repeticiones de los datos, las cuales fueron corregidas. A partir de este filtro se definen 354 datos viables (de 441 originales) para ser procesados, con lo cual se obtienen una nueva dirección preferencial de N50-60W para las fracturas del bloque Mojarra.

En Contreras (2008) se proponen sectores de interés hidrogeológico a través de mapas cualitativos de distribución espacial usando atributos de fractura relacionados con la apertura, la densidad y el buzamiento (Figura 2). La apertura se definió a partir de las direcciones de los esfuerzos de la zona asignando un carácter abierto o cerrado si las fracturas se encuentran más paralelas o perpendiculares al esfuerzo máximo; la densidad se categoriza en alta, media y baja según el espaciamiento promedio entre fracturas encontrado en campo sin especificar algún método de muestreo; finalmente Contreras (2008) separa los buzamientos tomados en campo en rangos desde $0^{\circ}$ hasta $90^{\circ}$. Estos mapas fueron utilizados para realizar un primer acercamiento a la zona y posteriormente compararlos con el soporte cuantitativo elaborado.

\section{Análisis cuantitativo del sistema de fractura}

Se presenta información de las 22 estaciones de campo donde se midieron datos de diaclasas: orientación (rumbo y buzamiento), apertura y descripciones complementarias. Además, se tomó registro fotográfico para el procesamiento. Los datos de diaclasas pertenecientes a las estaciones de la base de datos recolectada del estudio para aguas subterráneas realizado por INGEOMINAS y la Universidad Industrial de Santander (Pinto et al., 2007) sólo se tuvieron en cuenta para el cálculo de las orientaciones preferenciales. La ubicación de las estaciones de campo, tanto las provenientes de Pinto et al. (2007) como las del presente trabajo, se muestran en la Figura 1. Los resultados obtenidos se muestran en el mapa geológico de la zona en cinco bloques estructurales delimitados por fallas principales y que definen zonas, donde además las fallas secundarias muestran tendencias similares.

\section{Orientaciones preferenciales}

Orientaciones regionales: a través de FracPaQ se realizó el análisis a escala regional tomando como base el mapa de sombras generado a partir del modelo digital del terreno, sobre el cual se trazaron lineamientos estructurales en CorelDraw (siguiendo la metodología explicada antes). En este esquema se resaltaron las fallas geológicas ya identificadas en trabajos anteriores, especialmente las de Pinto et al. (2007). El resultado indica una tendencia NW con el vector que marca la media circular resultante en dirección N70W (línea roja a lo largo del gráfico, Figura 5).

Orientación de fracturas medidas en afloramientos: mediante el programa GeoRose 0.5.1 se determinaron tendencias de orientación preferencial a partir de la 
base de datos recolectada (Pinto et al., 2007) y los nuevos datos medidos en la campaña de campo, con un total de 551 planos de fractura. La Figura 6 muestra diagramas de rosa con las direcciones preferenciales de las fracturas del área de estudio y diagramas de densidad de polos que muestran predominios de inclinación de estos planos de fractura.

Los datos estructurales de toda la zona sur de la Mesa de Los Santos se muestran en diagrama de densidad de polos con predominio de altos grados de buzamiento, mientras que el diagrama de rosa indica una orientación preferencial de $\mathrm{N} 65 \mathrm{~W}$, similar a la obtenida anteriormente con FracPaQ. Por otro lado, se logró identificar predominio de altos grados de buzamiento en todos los cinco bloques, mientras que los diagramas de rosa muestran tres tendencias principales de orientación de fracturas: Los bloques 1 y 2 comparten una orientación preferencial cercana de
N60-70W y N70-80W respectivamente, concordantes con la orientación preferencial de toda el área y el rumbo regional de las fallas NW (fallas Potreros, Las Calaveras, La Mojarra, Los Santos y La Chivatera). El bloque 3 registra una orientación preferencial N20$30 \mathrm{~W}$, la cual concuerda con algunos trazos de fallas cortas que intersecan la Falla Zanjón La Vega. Al oeste, las orientaciones de los bloques 4 y 5 cambian de cuadrante presentando tendencias principales N0-10E y N10-20E, respectivamente, posiblemente representando fracturas ubicadas hacia los bordes de la mesa, generadas por liberación de energía o tensión en la cara libre de los escarpes. Otras direcciones secundarias se pueden notar en los bloques 2,3 y 4 . En el bloque 2 predomina un segundo conjunto de datos en dirección NE. En el bloque 3 una dirección casi vertical NS y en el bloque 4 se muestra una dirección N50-70W y N70-80E concordante con el trazo de las fallas NW y NE del mismo bloque.
A.

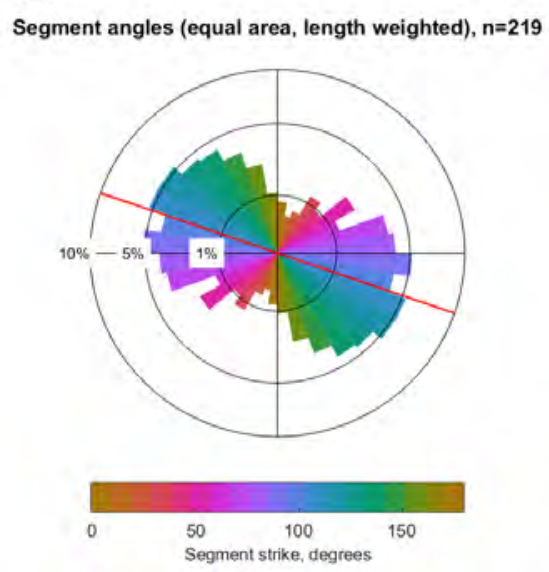

B.

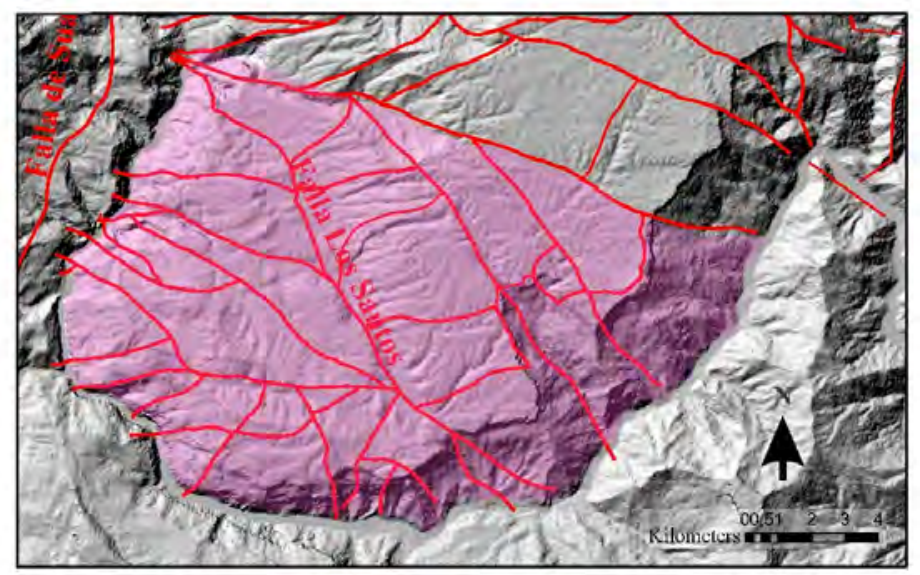

Figura 5. A. Diagrama de rosa regional, obtenido mediante FracPaQ a partir del trazo de lineamientos regionales. La línea roja a lo largo del diagrama de rosa indica la media circular resultante. B. Mapa de sombras utilizado para el procesamiento.

Orientación de fracturas en afloramientos a partir de registro fotográfico: se obtuvieron los diagramas de rosa de las 22 estaciones visitadas en campo a través de FracPaQ. De manera individual, cada uno de estos diagramas presenta dos tendencias de orientación preferencial de fracturas correspondientes a las diaclasas trazadas perpendiculares a los planos de estratificación y a las subparalelas a la estratificación (estas relaciones se establecen con las fotografías de campo y datos de estratificación tomados en campo).

Al analizar el gráfico resultante (Figura 7) se puede observar que las dos estaciones más cercanas a la Falla de Los Santos, YC005 y YC014, muestran tendencia hacia la orientación principal de la falla, aunque al sur
(YC014) predomine una tendencia ortogonal, es decir que se genera tensión asociada a la falla principal (la tensión es más propia de niveles superficiales). La gran mayoría de las estaciones cercanas a la Falla La Mojarra muestran un patrón similar, en donde la estación YC013 presenta como tendencia principal la ortogonal a la falla. Las estaciones YC021 y YC004 muestran una orientación W-E, aproximadamente a $60^{\circ}$ del trazo principal de la Falla La Mojarra. En el caso de las dos estaciones cercanas a la Falla Las Calaveras (YC015 y YC016) se identifica la tendencia hacia la orientación de la falla. Los diagramas de las estaciones YC009, YC018 y YC020, así como las tendencias paralelas a los escarpes de las demás estaciones localizadas en los bordes de la mesa, representan fracturas de tensión en la cara libre de estos afloramientos. 


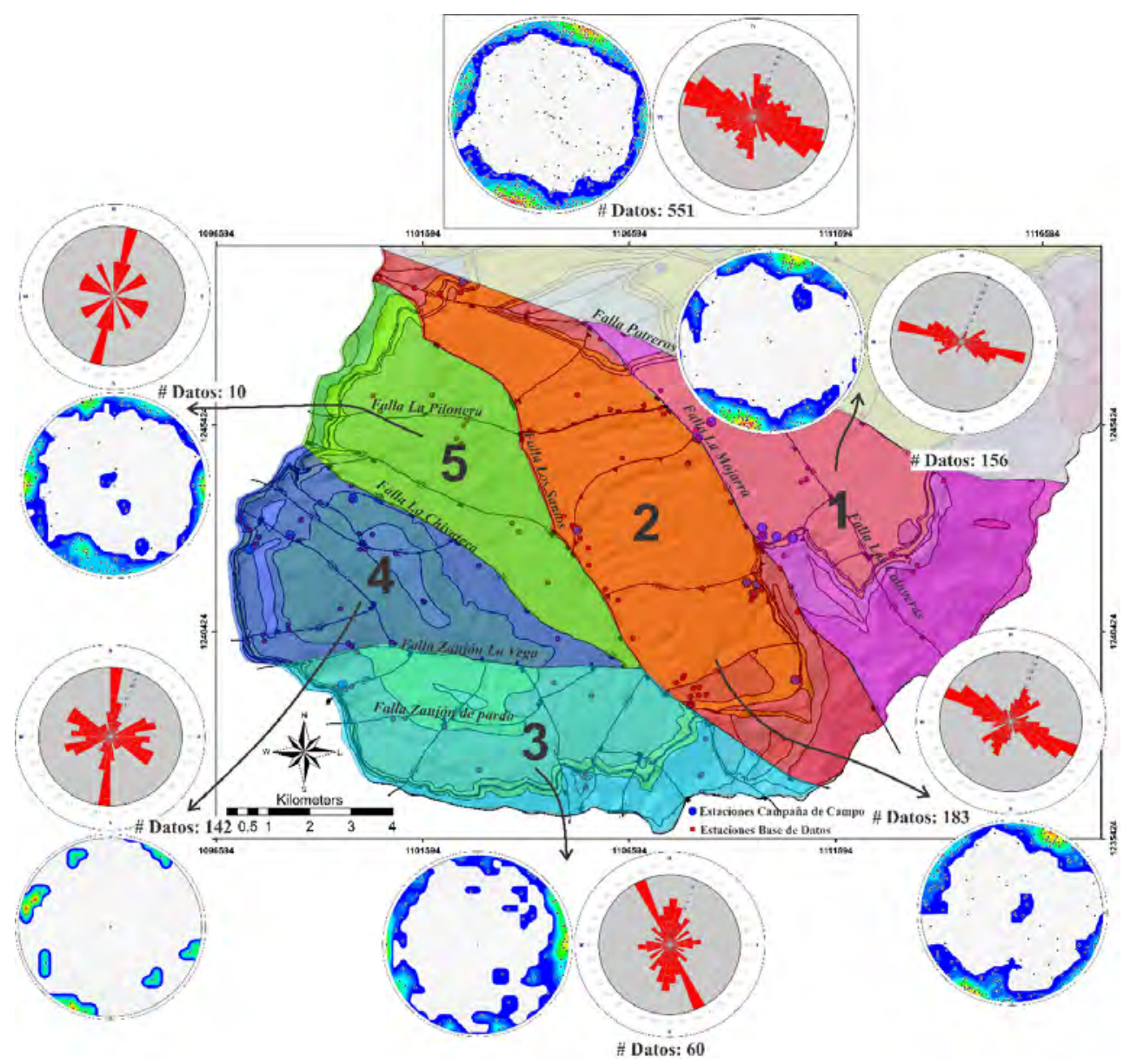

Figura 6. Diagramas de rosa y densidad de polos de los bloques estructurales. En la zona superior se muestra el diagrama de rosa y el diagrama de densidad de polos de todas las fracturas del área de estudio. En el mapa se ubican los mismos diagramas por bloques estructurales.

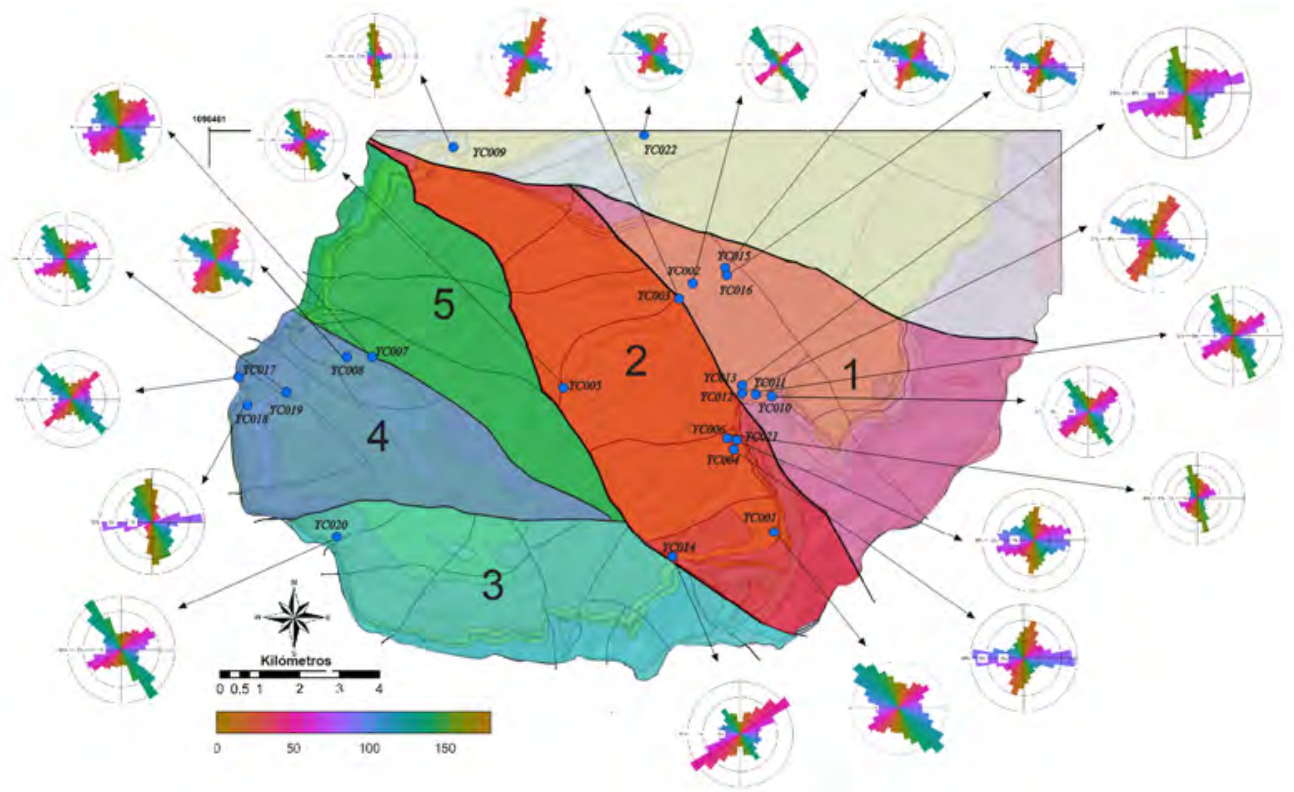

Figura 7. Mapa de diagramas de rosa por estación, obtenidos a partir de FracPaQ. 


\section{Apertura}

La escala mínima utilizada para identificar las aperturas en campo fue de 0,05 mm utilizando el comparador logarítmico de Ortega et al. (2006). Los resultados se proyectaron en un diagrama de dispersión en sentido NESW (Figura 8), siguiendo una orientación perpendicular a las fallas longitudinales las cuales se representan como líneas rojas verticales en dichos diagramas. $\mathrm{La}$ estación YC007 no se tuvo en cuenta pues se encuentra localizada en las laderas de la quebrada Chivatera y posiblemente la acción erosiva de la corriente de agua contribuyó en el grosor de la abertura.
Las aperturas más significativas se encontraron en las estaciones YC013, YC017, YC003, YC021 y YC022 pertenecientes al miembro superior de la Formación Los Santos asociadas a las fallas NW de la zona (Los Santos y La Mojarra) (Figura 7). Generalmente las menores aperturas se encuentran en zonas distanciadas de fallas; un ejemplo de esto es la correlación de un grupo de estaciones ubicadas al noreste de la Falla La Mojarra desde la estación YC010 (estación más lejana a la falla) hasta la estación 13, en la cual se observa un aumento progresivo en el valor de la apertura al acercarse a su trazo regional.

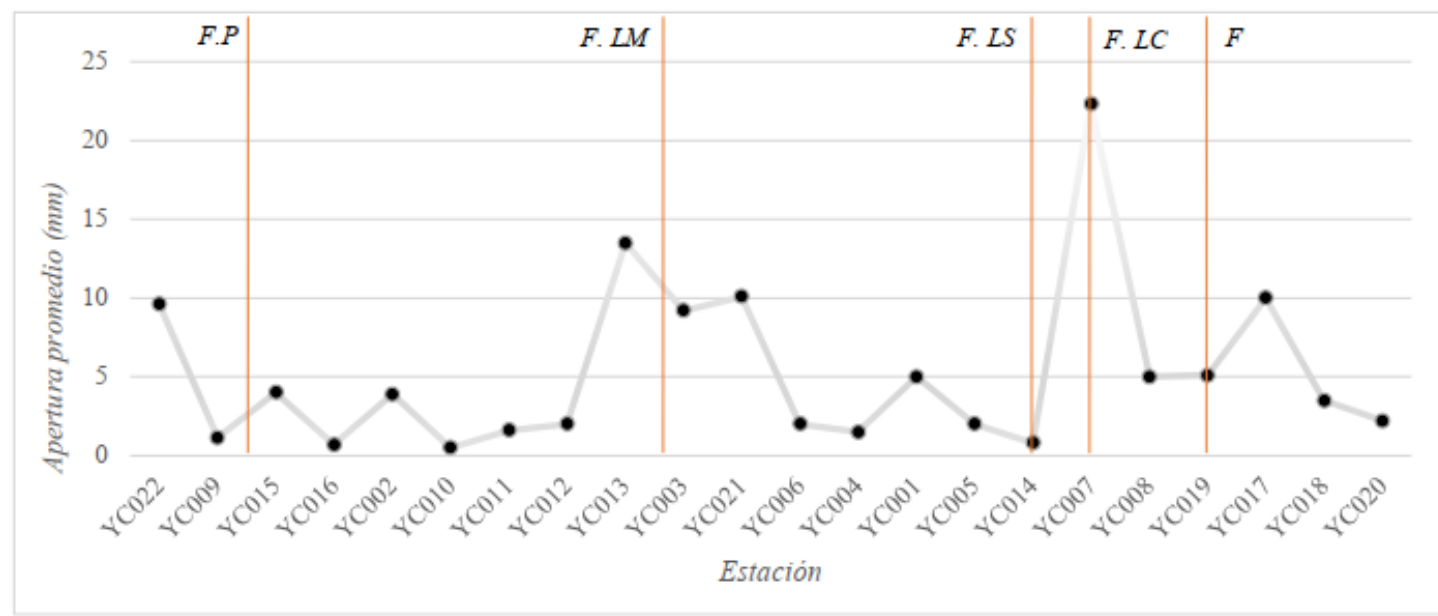

Figura 8. Gráfico de dispersión de la apertura. F: Falla, F. P: Falla Potreros, F. LM: Falla La Mojarra, F.LS: Falla Los Santos, F.LC: Falla La Chivatera. Debido a la linealidad del diagrama, no es posible escalar las distancias reales entre las estaciones y las fallas, es por esto que para mayor comprensión espacial se recomienda revisarlo junto con el mapa de fallas y estaciones (Figura 1).

\section{Intensidad, densidad y longitud de fractura}

A través de FracPaQ se estimaron la intensidad (Figura 9), densidad (Figura 10) y longitud promedio de los patrones de fractura. Los rectángulos delimitadores se grafican por estación y se clasifican en un rango comparativo de bajo, medio, alto y muy alto según los valores obtenidos. En estos rectángulos se muestran los principales patrones de fractura, así como las más pequeñas presentes en cada afloramiento. Los resultados cuantitativos se muestran en la Tabla 1.

Tabla 1. Medidas de intensidad, densidad y longitud promedio.

\begin{tabular}{cccc}
\hline Estación & Intensidad $\left(\mathbf{m}^{-\mathbf{1}}\right)$ & Densidad $\left(\mathbf{m}^{-\mathbf{2}}\right)$ & Longitud promedio $(\mathbf{c m})$ \\
\hline YC001 & 38,19 & 2535,40 & 3 \\
YC002 & 34,38 & 1216,55 & 9 \\
YC003 & 32,59 & 1237,11 & 7 \\
YC004 & 18,18 & 510,48 & 12 \\
YC005 & 27,46 & 1405,54 & 5 \\
YC006 & 49,67 & 6064,11 & 3 \\
YC007 & 11,95 & 289,81 & 14 \\
YC008 & 24,34 & 570,64 & 10 \\
YC009 & 21,66 & 1299,92 & 7 \\
\hline
\end{tabular}


Continuación Tabla 1.

\begin{tabular}{cccc}
\hline Estación & Intensidad $\left(\mathbf{m}^{-\mathbf{1}}\right)$ & Densidad $\left(\mathbf{m}^{-\mathbf{2}}\right)$ & Longitud promedio $(\mathbf{c m})$ \\
\hline YC010 & 52,95 & 2389,00 & 5 \\
YC011 & 43,63 & 2336,52 & 6 \\
YC012 & 25,05 & 922,00 & 12 \\
YC013 & 33,31 & 2131,55 & 6 \\
YC014 & 18,36 & 353,08 & 13 \\
YC015 & 18,59 & 790,68 & 7 \\
YC016 & 27,23 & 536,87 & 13 \\
YC017 & 34,45 & 3548,95 & 5 \\
YC018 & 15,85 & 262,46 & 14 \\
YC019 & 13,66 & 631,72 & 7 \\
YC020 & 11,07 & 129,51 & 19 \\
YC021 & 20,71 & 842,25 & 7 \\
YC022 & 20,95 & 356,28 & 13 \\
\hline
\end{tabular}

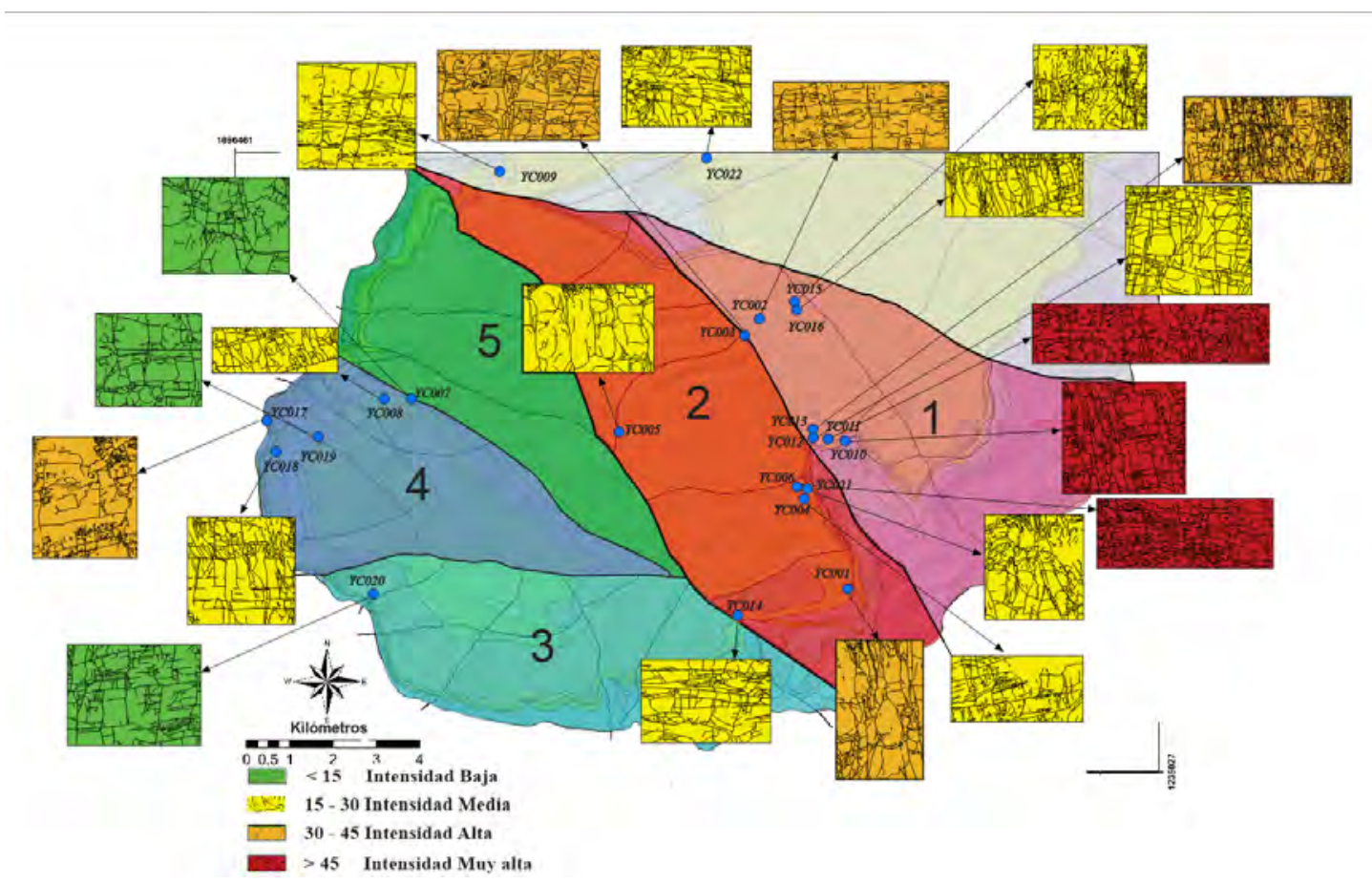

Figura 9. Mapa de intensidades del área por estaciones de estudio.

Al sur de la Mesa de Los Santos las zonas que presentan mayor fracturamiento están asociadas a las fallas longitudinales NW, especialmente en el miembro superior de la Formación Los Santos en el área de influencia de la Falla La Mojarra, donde se registran los valores más altos de intensidad y densidad (Tabla 1). Al NE de esta falla se encuentra también la estación YC006 con el mayor valor de densidad encontrado de $6064 \mathrm{~m}^{-2}$ y la estación YC001 de densidad $2535 \mathrm{~m}^{-2}$. Por otro lado, el menor valor se registra al suroeste, asociada a la Formación Rosablanca, en la estación YC020 $\left(129,51 \mathrm{~m}^{-2}\right)$; así como en otras estaciones ubicadas al suroeste de la zona de estudio que también presentan las estimaciones más bajas de intensidad. Otras densidades significativas se detectaron en los cruces de fallas donde se encuentran las estaciones YC003, YC006 y YC005. Los valores más altos de densidad e intensidad se presentan en el miembro superior de la Formación Los Santos constituido mayormente por areniscas cuarzosas. 


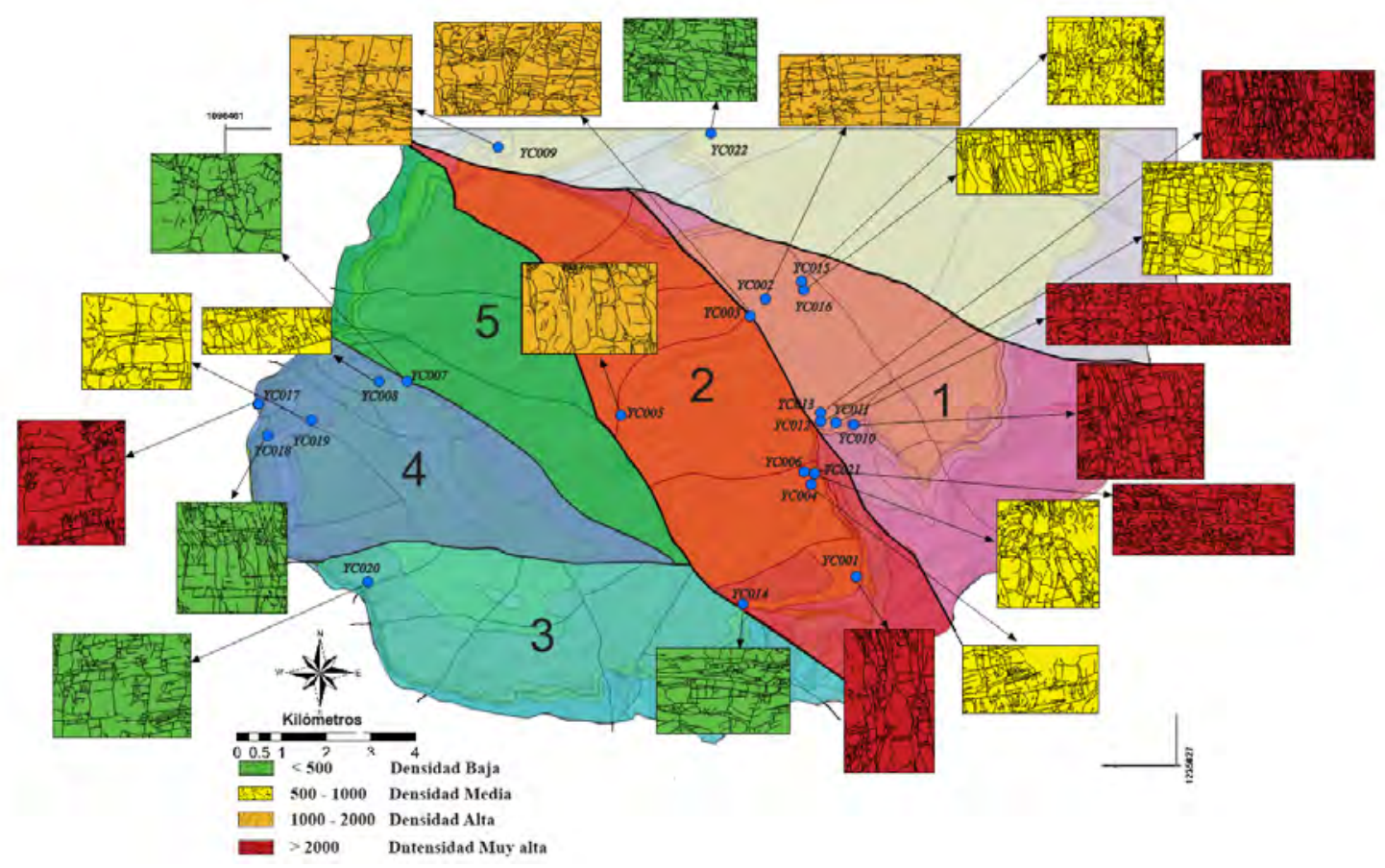

Figura 10. Mapa de densidades del área por estaciones de estudio.

Al igual que la apertura, los resultados de longitud fueron organizaron en un diagrama de dispersión (Figura 11) en sentido NE-SW, siguiendo una orientación perpendicular a las fallas longitudinales las cuales se representan como líneas rojas verticales en dichos diagramas; y como se menciona anteriormente, se recomienda revisarlo junto con el mapa de fallas y estaciones (Figura 1) para mayor comprensión. Se observa que en general la longitud promedio de las fracturas es menor a $20 \mathrm{~cm}$. Las medidas más largas se atribuyen a las diaclasas con trazos verticales y las más cortas son asociadas a fracturas subparalelas a la estratificación. Las estaciones con mayor densidad (por ejemplo, YC006, YC010, YC017) presentan las longitudes de traza promedio más cortas en comparación con las estaciones de más baja densidad (por ejemplo, YC007, YC020, YC018) que tiene longitudes promedio de $14 \mathrm{~cm}$ y $19 \mathrm{~cm}$. Es importante señalar que las medidas de longitud promedio están confinadas a una capa o estrato individual del afloramiento que a la vez se restringen a las líneas de fractura trazadas en el rectángulo delimitador con un nivel de zoom significativo, es decir se tomaron longitudes de milímetros y centímetros.

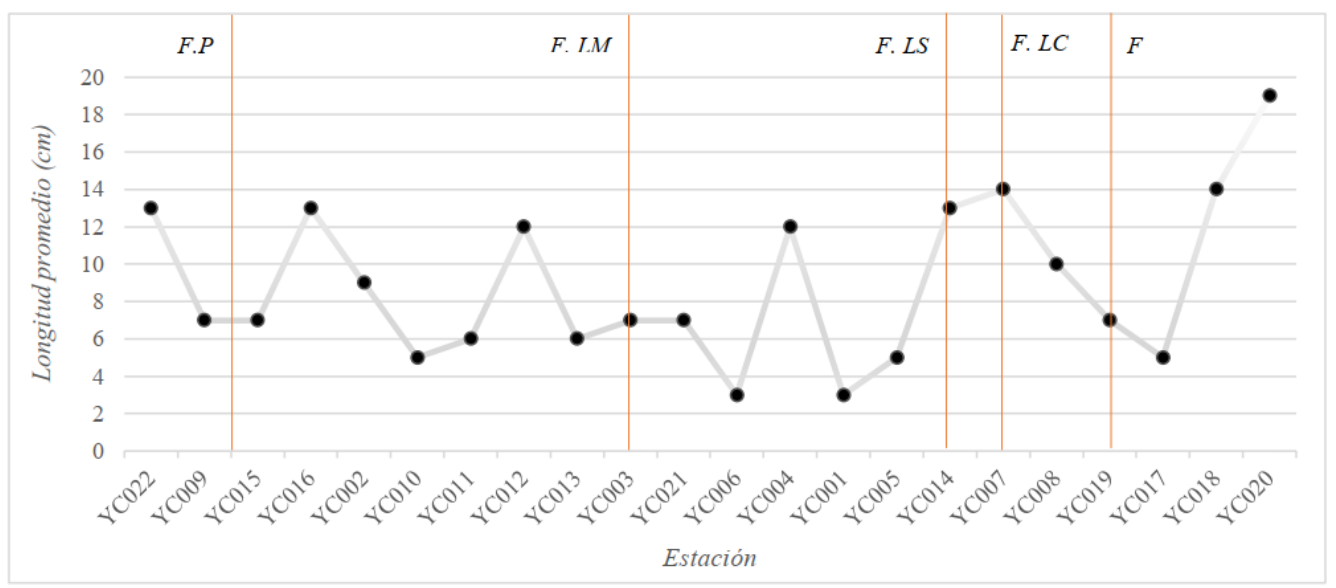

Figura 11. Gráfica de dispersión de la longitud de traza promedio. F: Falla, F. P: Falla Potreros, F. LM: Falla La Mojarra, F.LS: Falla Los Santos, F.LC: Falla La Chivatera. 


\section{Análisis preliminar del flujo de fluidos: conectividad y permeabilidad}

Los resultados de este análisis son significativos en caso de que se garantice la presencia de un fluido transportado a través de las diferentes estructuras. Preliminarmente el flujo de fluidos se determina mediante el gráfico ternario de conectividad propuesto por Manzocchi (2002) y la orientación de la elipse del tensor de permeabilidad en dirección del flujo.

De manera general, las redes más conectadas se trazan hacia la línea Y-X del grafico ternario de conectividad ubicada en la parte inferior, mientras que las redes menos conectadas se trazarán hacia el vértice $\mathrm{I}$. Para evaluar la conexión por línea (CL) se toma de referencia las líneas de conectividad de contorno para CL $=2$ y 3,57, donde los resultados mayores a 2 contaran con mejores conectividades. Por otra parte, la estimación de la permeabilidad se realizó a través del tensor de fractura con la elipse de permeabilidad en dirección del flujo, donde el eje principal (orientación en la cual se alarga) está en la dirección de máxima permeabilidad $(\mathrm{k} 1)$, es decir, indica el camino más probable que favorecería el flujo de un potencial fluido a través de la red de fracturas. Para ejemplificar esta relación se presentan los gráficos resultados de la estación YC012 ubicada espacialmente en el bloque 1 y asociada estratigráficamente al miembro superior de la Formación Los Santos (Figura 12). Los resultados de conectividad y permeabilidad de cada estación se muestran en la Figura 13.
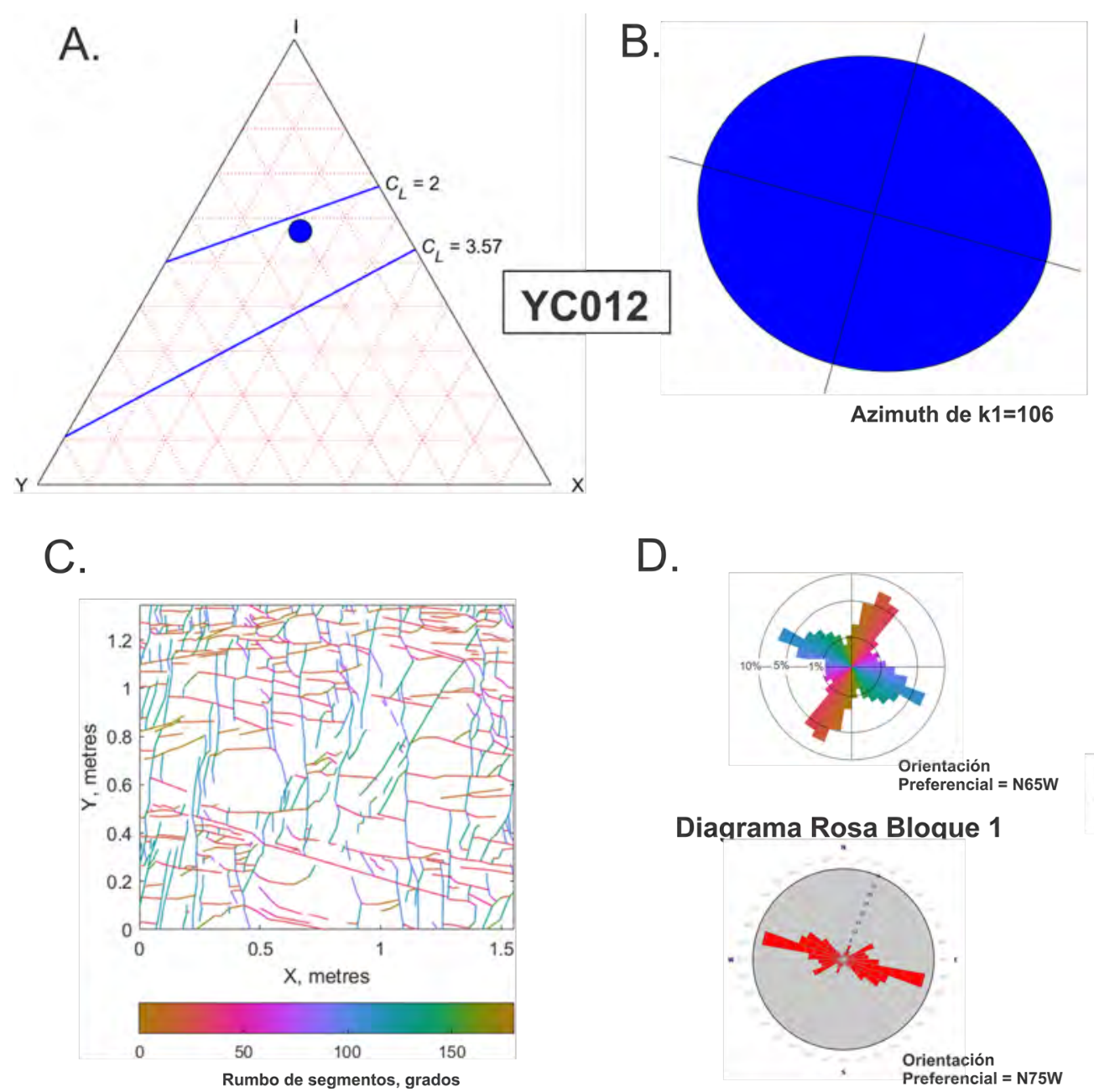

Figura 12. Relación entre gráficos de la estación YC12. A. Gráfico ternario de conectividad para la estación YC012. B. Permeabilidad en dirección del flujo de la estación YC012, kij describe un tensor de permeabilidad de segundo orden para el flujo de fluido a través de la red de fracturas. C. Mapa de rumbos de segmento a partir de la digitalización del registro fotográfico de la estación YC012. D. Diagrama de rosa de la estación YC012 y diagrama de rosa del bloque 1. Estas características son comparables con las demás estaciones para el bloque 1 , donde se presentan las mejores condiciones de conectividad y permeabilidad asociada a mayores buzamientos que permitirían la infiltración a mayores profundidades. 
Para el bloque 1, la mayoría de los gráficos ternarios muestran una conexión por línea mayor a 2 con dirección del flujo orientada al NW-SE concordante con la orientación preferencial de las diaclasas obtenidas para este bloque y relacionadas en el registro fotográfico con las fracturas más extensas y de mayor buzamiento $\left(80^{\circ}-90^{\circ}\right)$ encontradas en el afloramiento (Figura
12). Para el bloque 2, la dirección de permeabilidad cambia a NE-SW mostrando una relación más cercana a las fracturas subparalelas a la estratificación. La conexión por línea es variable, con valores cercanos y en algunos casos superiores a 2 En este bloque se observan fallas en sentido E-W que pueden influir en el comportamiento de la permeabilidad.

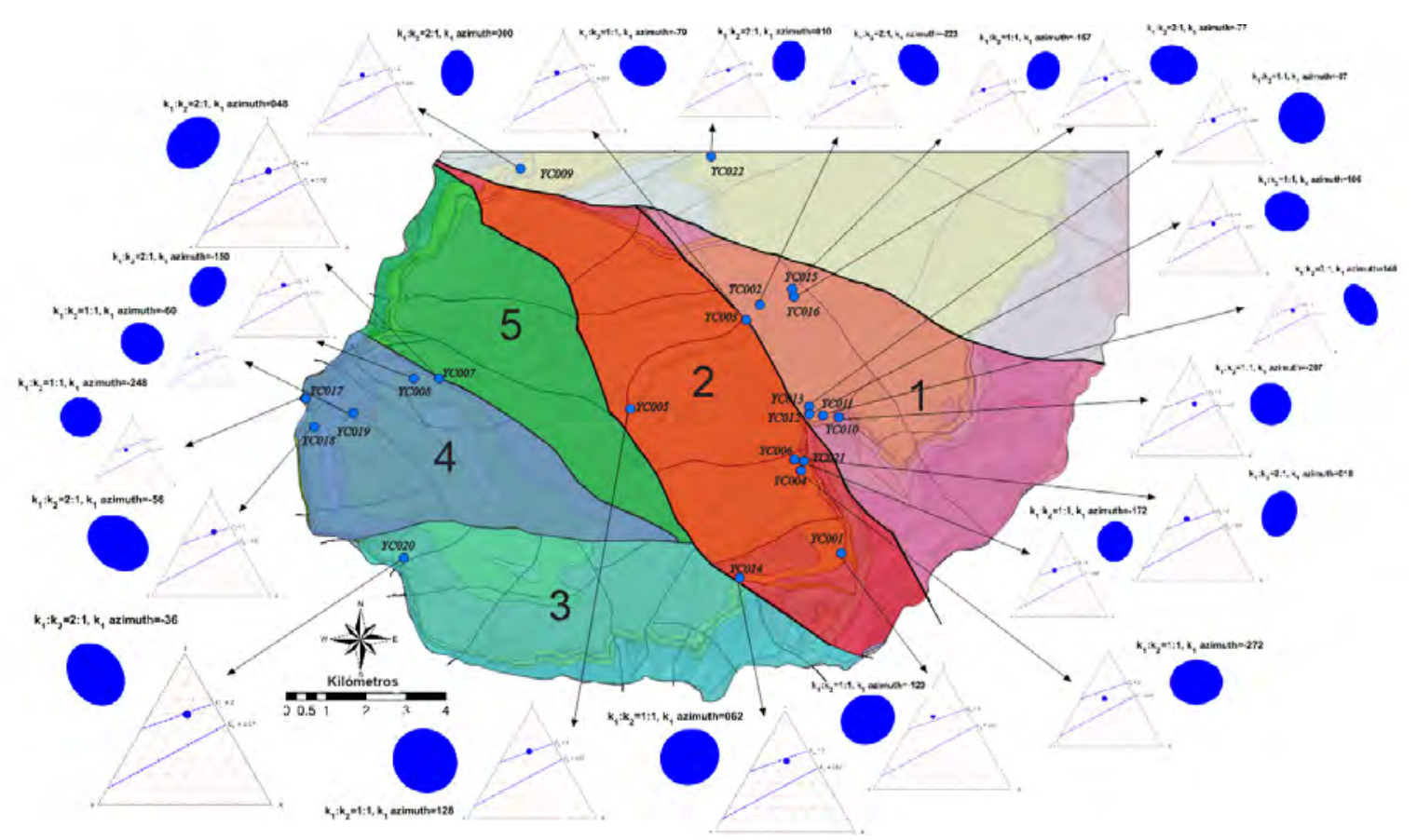

Figura 13. Mapa de conectividades y permeabilidades del área por estaciones de estudio.

Las elipses de permeabilidad en dirección del flujo de las estaciones pertenecientes al bloque 3 y 4 comparten una dirección de permeabilidad máxima orientada al NW-SE, concordante con la orientación preferencial de las diaclasas para el bloque 3 (N20-30W). Sin embargo, las estaciones cercanas a la Falla La Chivatera (YC007 y YC008) cambian a una dirección NE-SW. El área asociada a la Formación Rosablanca presenta la menor conexión por línea con un promedio menor a 2 , que indica las condiciones menos favorables de conectividad. Según la mayoría de inclinaciones observadas en diagramas de polos $\left(80^{\circ}-90^{\circ}\right)$ (Figura 6), pueden presentarse condiciones favorables para mantener la conectividad en profundidad y por lo tanto posibilidades de acuíferos fracturados si hay suficiente recarga.

\section{Interpretación y discusión}

Como marco estructural preexistente para esta interpretación y discusión se consideran los trabajos locales de Pinto et al. (2007), Velandia et al. (2007), Vargas
(2008), Contreras (2008), Díaz et al. (2009) y Velandia (2010), así como los de carácter regional enfocados a definir un tensor de esfuerzos que explique la cinemática de las fallas (Velandia y Bermúdez, 2018; Velandia et al., 2020), incluidas las estructuras de la Mesa de Los Santos. El análisis se presenta aquí según la localización de las estaciones de campo respecto de las principales fallas y de los bloques definidos por los autores.

Las estaciones asociadas a la Falla La Mojarra registran los valores más altos de densidad e intensidad de fracturas, en consecuencia, se puede estimar una mayor porosidad secundaria asociada al miembro superior de la Formación Los Santos. Es importante señalar que la estación YC017 ubicada al suroeste y relacionada al trazo NW y antitético de la Falla Zanjón La Vega, presenta una densidad muy alta asociada al miembro superior de la Formación Los Santos.

En comparación con el mapa de distribución espacial de la densidad de fracturamiento obtenido por Contreras (2008) se observa una buena correlación 
espacial en los rangos obtenidos de alta, media y baja densidad. Dada esta compatibilidad se confirma el modelo estructural previo (Contreras, 2008; Díaz et al., 2009) que además se plasmó en los estudios base para el modelo hidrogeológico conceptual y preliminar de INGEOMINAS y UIS (Pinto et al., 2007). Así se hace énfasis en la zona de influencia de la Falla La Mojarra como el área más favorable para la infiltración y flujo subsuperficial. Llama la atención su conexión con la Falla Potreros, límite norte de nuestra zona de estudio, por tratarse de una estructura transtensiva (Velandia et al., 2007; Pinto et al., 2007; Vargas, 2008) que también podría facilitar el flujo.

Aunque el suroeste de la zona no muestra un fracturamiento significativo, las trazas de las fallas Los Santos y La Chivatera podrían afectar el miembro superior de la Formación Los Santos, al igual que la Falla La Mojarra, ya que comparten orientación y cinemática. Por lo que se propone estudiar más profundo estas zonas de daño para establecer posibles corredores. Preliminarmente, estas estructuras de rumbo NW pueden considerarse como potenciales corredores para el tránsito y acumulación de fluidos ya que se disponen en forma subparalela con la orientación del esfuerzo máximo horizontal (tensor de esfuerzos), que en la zona explica la cinemática sinestral de la Falla de Bucaramanga (Velandia y Bermúdez, 2018).

Las gráficas de conectividad del área de estudio (Figura 13) son similares, sin embargo, las estaciones que se localizan en el miembro superior de la Formación Los Santos cuentan con una proporción ligeramente mayor de nodos "X" y una proporción menor de nodos "I" que las restantes estaciones (Figura 13), lo que sugiere una mayor conectividad en general, datos que de forma más cuantitativa corrobora resultados previos de Contreras (2008) y de Díaz et al. (2009). La permeabilidad estimada muestra que el azimut k1 (dirección de permeabilidad en dirección del flujo) para la mayoría de estaciones al norte de la Falla La Mojarra es paralelo al conjunto de fracturas largas de alta densidad en los afloramientos, mientras que al sur de la misma (Figura 13), la mayoría de conjuntos de datos de azimut $\mathrm{k} 1$ de las estaciones está orientado en forma paralela al conjunto más corto y menos denso de fracturas. Este cambio en el comportamiento de la permeabilidad podría ser debido a la Falla La Mojarra, la cual se comportaría como corredor para los acuíferos adyacentes, favoreciendo más el flujo local. Otras estructuras que pueden explicar los valores asociados con corredores de flujo son las fallas orientadas W-E.
Otro tipo de consideración observable para el análisis de estas zonas destacables de fractura es interpretar las interconexiones entre fracturas a profundidad, que están dadas por las estaciones con ángulos bajos, en este caso el bloque 2 presenta un mayor número de fracturas con ángulo medio a bajo que los otros bloques. Las áreas donde hay cruces de fallas y estaciones en bordes de escarpe también son favorables para la interconexión.

Según el esquema hidrogeológico preliminar (Hincapié y Veloza, 2009) la recarga ocurre a lo largo de sistemas de fracturas a partir de la precipitación desde los sectores topográficamente más altos ubicados al NE de la mesa. Los resultados del presente trabajo muestran la gran mayoría de las estaciones localizadas en la zona de interés hidrogeológico establecida o zona influenciada por la Falla La Mojarra (por presentar los valores más altos de intensidad/densidad) favorecen la recarga del sistema acuífero, corroborando el planteamiento de Pinto et al. (2007) y de Hincapié y Veloza (2009) indicando una recarga del acuífero más superficial (acuífero libre) presente en el miembro superior de la Formación Los Santos, el cual corresponde a areniscas que localmente presentan espesos perfiles de meteorización que permiten además la recarga difusa.

Dada la alta inclinación de la mayoría de las fracturas no se puede descartar que la recarga llegue a los acuíferos más profundos. A partir de los resultados de permeabilidad se analizó si el flujo en efecto podría transcurrir en forma superficial o profunda. Algunas presentan el azimut k1 (dirección de permeabilidad) paralelo a la orientación del conjunto de fracturas dispuestas en forma subortogonal a la estratificación, es decir que el flujo tendería a profundizarse. En otras estaciones se presenta un azimut k1 paralelo a la orientación del conjunto de fracturas subparalelas a la estratificación, sin embargo, la inclinación de los estratos en la mayoría de estas estaciones es hacia el oeste, es decir que el flujo correría hacia el interior de la Mesa de Los Santos.

\section{Conclusiones}

El análisis cuantitativo al sistema de fracturas al sur de la Mesa de Los Santos se realizó mediante los programas FracPaQ y Georose, con el fin de determinar la abundancia y variación espacial de las fracturas en el área de estudio. A partir de datos digitales se obtuvieron resultados congruentes con investigaciones previas que permiten abrir una discusión sobre las 
implicaciones hidrogeológicas de las estructuras para un estudio más integral del agua subterránea en la Mesa de Los Santos.

Por medio del análisis de 551 planos de diaclasa se determinó N60-70W como la orientación preferencial de las fracturas en la zona sur de la Mesa de Los Santos. La cuantificación de los patrones de fractura y el análisis preliminar del flujo de fluidos (conectividad y permeabilidad) de las 22 estaciones visitadas apuntaron a zonas de interés hidrogeológico que podrían favorecer la recarga del sistema acuífero y a establecer posibles interconexiones a profundidad relacionadas con los bajos a intermedio ángulos de fracturas.

Según su orientación, las fallas NW se destacan por su apertura, intensidad y densidad (criterios fundamentales para identificar posibles zonas de infiltración y flujo subsuperficial) de las fracturas aledañas. La Falla La Mojarra afecta al miembro superior de la Formación Los Santos al NE del área de estudio, y se asocia con los valores de intensidad y densidad más altos ( $>45$ $\mathrm{m}^{-1} \mathrm{y}>2000 \mathrm{~m}^{-2}$ respectivamente), así como al control en el grosor de la apertura de las fracturas, lo que permite catalogar esta estructura y su zona de daño como la más favorable para la posible infiltración y almacenamiento de fluidos.

Las zonas de alto interés hidrogeológico, identificadas a partir de la metodología usada y el conocimiento hidrogeológico previo del área que fueron base para este trabajo, contribuyen al conocimiento de las estructuras geológicas que rigen el comportamiento del sistema, importantes para la construcción de un modelo hidrogeológico conceptual y el estudio de flujo de fluidos de aguas subterráneas en la Mesa de Los Santos.

\section{Agradecimientos}

Este manuscrito incluye parte de los resultados del trabajo de grado de Tarazona y Vargas y corresponde al tema de geología estructural del proyecto de investigación 2534 de la Vicerrectoría de Investigación y Extensión de la UIS, denominado "Estudio integral del agua en la Mesa de Los Santos”. Gracias al apoyo de los grupos de investigación GIGBA y GPH, así como de los habitantes del municipio de Los Santos por su hospitalidad y guía en el camino. Nuestro agradecimiento a Óscar García-Cabrejo y a un segundo revisor anónimo por sus observaciones y correcciones, las cuales nos permitieron dar más claridad al manuscrito.

\section{Referencias}

Arguello, A.; Becerra, N.; Herrera, L.; Prada, F. (2018). Estudio del recurso hídrico subterráneo en la zona sur occidental del municipio de Los Santos, Santander. Tesis de pregrado. Universidad Industrial de Santander, Bucaramanga, Colombia.

Clavijo, J.; Royero, J.M. (2001). Mapa geológico generalizado departamento de Santander. Escala 1:300.000. Memoria explicativa. Instituto Nacional de Investigaciones Geológico Mineras (INGEOMINAS).

Contreras, N. (2008). Análisis del fracturamiento presente en la Formación Los Santos al noreste y centro de la Mesa de Los Santos, departamento de Santander. Tesis de pregrado. Universidad Industrial de Santander, Bucaramanga, Colombia.

Díaz, E.; Contreras, N.M.; Pinto, J.E.; Velandia, F.; Morales, C.J.; Hincapie, G. (2009). Evaluación hidrogeológica preliminar de las unidades geológicas de la Mesa de los Santos, Santander. Boletín de Geología, 31(1), 61-70.

Healy, D.; Rizzo, R.E.; Cornwell, D.G.; Farrell, N.J.C.; Watkins, H.; Timms, N.E.; Gomez-Rivas, E.; Smith, M. (2017). FracPaQ: A MATLAB ${ }^{\mathrm{TM}}$ toolbox for the quantification of fracture patterns. Journal of Structural Geology, 95, 1-16. https:// doi.org/10.1016/j.jsg.2016.12.003

Healy, D.; Rizzo, R. (2019). FracPaQ: Fracture pattern quantification. User guide version 2.6. https:// github.com/DaveHealy-github/FracPaQ

Hincapié, G.; Veloza, J. (2009). Informe hidrogeológico de la Mesa de Los Santos. Bogotá. Instituto Colombiano de Geología y Minería (INGEOMINAS).

Julivert, M. (1958). La morfoestructura de la zona de las mesas al SW de Bucaramanga (Colombia S. A.). Boletín de Geología, 1, 7-43.

Manzocchi, T. (2002). The connectivity of twodimensional networks of spatially correlated fractures. Water Resources Research, 38(9), 1-20. https://doi.org/10.1029/2000WR000180

Mauldon, M.; Dunne, W.M.; Rohrbaugh, M.B. (2001). Circular scanlines and circular windows: New tools 
for characterizing the geometry of fracture traces. Journal of Structural Geology, 23(2-3), 247-258. https://doi.org/10.1016/S0191-8141(00)00094-8

Morales, C.J.; Forero, H.; Rojas, N.; Alvarado, S. (2010). Integración geológica e hidrogeológica del centro de Santander. Bogotá. Instituto Colombiano de Geología y Minería (INGEOMINAS).

Moreno-Sánchez, G.; García-Cabrejo, O. (2006). Caracterización cuantitativa de patrones de fracturamiento mediante ventanas circulares y análisis fractal. Geología Colombiana, 31, 73-90.

Moreno, M.L.; Silva, K. (2021). Identificación de ambientes geomorfológicos y elaboración de un mapa de favorabilidad para la percolación de la zona de la Mesa de Los Santos, Santander. Tesis de grado. Universidad Industrial de Santander, Bucaramanga, Colombia.

Ortega, O.J.; Marrett, R.A.; Laubach, S.E. (2006). A scale-independent approach to fracture intensity and average spacing measurement. AAPG Bulletin, 90(2), 193-208. https://doi. org/10.1306/08250505059

París, G.; Machette, M.N.; Dart, R.L.; Haller, K.M. (2000). Map and Database of Quaternary Faults in Colombia and its Offshore Regions. U.S. Geological Survey Open-File Report 00-018 (1:2.000.000 scale). https://doi.org/10.3133/ofr00284

Pinto, J.E.; Clavijo, J.; Gómez, S.; Gutiérrez, D.; Mora, J.P.; Rojas, N.; Adarme, I.; Aldana, S.; Contreras, N.; Díaz, E.; Melo, L.; Reátiga, G.; Rincón, M.; Vargas, C.; Morales, C.; Velandia, F.; Hincapié, G.; Forero, H.; Duarte, R.; De Bermúdez, O. (2007). Proyecto de investigación geológica e hidrogeológica en la Mesa de Los Santos, sector noreste de Curití y borde occidental del macizo de Santander, departamento de Santander. Bucaramanga. Instituto Colombiano de Geología y Minería (INGEOMINAS).

Siravo, G.; Fellin, M.G.; Faccena, C.; Maden, C. (2020). Transpression and build-up of the cordillera: the example of the Bucaramanga fault (eastern cordillera, Colombia). Journal of the Geological Society, 177(1), 14-30. https://doi.org/10.1144/ jgs2019-054
Taboada, A.; Rivera, L.; Fuenzalida, A.; Cisternas, A.; Philip, H.; Bijwaard, H.; Olaya, J.; Rivera, C. (2000). Geodynamics of the northern Andes: Subductions and Intracontinental deformation (Colombia). Tectonics, 19(5), 787-813. https://doi. org/10.1029/2000TC900004

Tarazona, Y.; Vargas, M.C. (2020). Análisis cuantitativo de sistemas de fracturas como aporte al conocimiento hidrogeológico de la zona sur de la Mesa de Los Santos, Santander - Colombia. Tesis de pregrado. Universidad Industrial de Santander, Bucaramanga, Colombia.

Vargas, C.Y. (2008). Cartografía geológica a escala 1:25.000 de la Mesa de Los Santos, departamento de Santander. Tesis de pregrado. Universidad Industrial de Santander, Bucaramanga, Colombia.

Velandia, F.; Silva, G.; Morales, C.; Osorio, J. (2007). Análisis cinemático de la región central del departamento de Santander. Memorias. XI Congreso Colombiano de Geología, Bucaramanga, Colombia.

Velandia, F.A. (2010). Análisis de fracturas para exploración de aguas subterráneas en el centro de Santander. En: Hidrogeología para la gestión del recurso hídrico (pp. 149-158). Universidad de Antioquia.

Velandia, F.; Bermúdez, M. (2018). The transpressive southern termination of the Bucaramanga fault (Colombia): insights from geological mapping, stress tensors, and fractal analysis. Journal of Structural Geology, 115, 190-207. https://doi. org/10.1016/j.jsg.2018.07.020

Velandia, F.; García-Delgado, H.; Zuluaga, C.A.; López, J.A.; Bermúdez, M.A.; Audemard, F.A. (2020). Present-day structural frame of the Santander Massif and Pamplona Wedge: The interaction of the Northern Andes. Journal of Structural Geology, 137. https://doi.org/10.1016/j.jsg.2020.104087

Yong Technology Inc. (2015). GeoRose 0.5.1. A free geological Rose diagram and stereonet plotting program. Edmonton, Canada. http://www. yongtechnology.com/georose/

Fecha de recibido: 21 de julio de 2021

Fecha de aprobado: 21 de septiembre de 2021 